\title{
11 \\ Rudolf Carnap und Kurt Gödel: Die beiderseitige Bezugnahme in ihren philosophischen Selbstzeugnissen
}

\author{
Eva-Maria Engelen
}

\subsection{Einleitung}

In diesem Beitrag wird die gegenseitige Wahrnehmung und Einflussnahme von Rudolf Carnap und Kurt Gödel aufeinander auf Grund der von ihnen erhaltenen jeweiligen philosophischen Selbstzeugnisse während der 20er- bis 40er-Jahre des 20. Jahrhunderts untersucht. Unter diese Selbstzeugnisse fallen zum einen Carnaps Tagebücher, seine Gesprächsnotizen und der Briefwechsel mit Gödel, zum anderen Gödels philosophische Notizbücher. Das sich daraus ergebende Bild erlaubt es, die bisherige Forschung zum Einfluss der beiden Denker aufeinander zu bestätigen, zu vervollständigen sowie neue Aspekte aufzudecken.

Schon jetzt bieten Carnaps Tagebücher von 1926 bis 1935, seine Gesprächsnotizen, der Briefwechsel zwischen ihm und Gödel sowie die philosophischen Notizbücher Gödels aufschlussreiches Material über den geistigen Austausch der beiden in den gemeinsamen Wiener Jahren. Beide Denker nehmen sich in dieser Zeit gegenseitig vornehmlich als mathematische Logiker wahr und weniger als breit gefächerte Philosophen. Bei Gödel hat, wie ein Eintrag im Notizbuch Max Phil XI explizit zeigt, im Laufe der Jahre in der Wahrnehmung Carnaps eine Veränderung stattgefunden. Er setzt sich spätestens ab 1934 mit Carnap als einem Philosophen auseinander, für den die Logik grundlegend für die Philosophie ist, die Philosophie aber im Vordergrund steht. Carnap hingegen konsultiert Gödel vornehmlich als jemanden, der ihm helfen kann, seine logischen Probleme zu lösen. Carnaps Einträge vom 16. März 1930 und 12. September 1930 (s. u.) legen zwar nahe, dass Carnap durchaus philosophische Stellungnahmen Gödels zur Kenntnis genommen hat, und man kann sogar nachweisen, dass er durch sie beeinflusst wurde, aber Carnap be-

E.-M. Engelen ( $\square)$

Berlin-Brandenburgische Akademie der Wissenschaften, Berlin, Deutschland

E-Mail: eva-maria.engelen@bbaw.de

(C) The Author(s) 2021

C. Damböck, G. Wolters (eds.), Der junge Carnap in historischem Kontext:

1918-1935 / Young Carnap in an Historical Context: 1918-1935,

Veröffentlichungen des Instituts Wiener Kreis 30,

https://doi.org/10.1007/978-3-030-58251-7_11 
zieht sich hinsichtlich der Diskussionen mit Gödel jeweils immer nur auf einzelne, ihm wichtige Punkte, nie auf Gödel als einen Denker, der eine eigene philosophische Auffassung begründen könnte.

Der vorliegende Vergleich muss bedauerlicherweise auf die Transkription von Carnaps Tagebüchern von 1926 bis 1935 beschränkt werden, da die folgenden Jahrgänge noch nicht transkribiert sind. Insbesondere die Jahrgänge 1952-1954 von Carnaps Tagebüchern wären von großem Interesse. Sie betreffen die beiden Jahre, in denen Carnap und Gödel gleichzeitig in Princeton waren und sich, wie wir aus Carnaps ungekürzter Autobiographie wissen, des Öfteren getroffen haben. Zum Inhalt dieser Gespräche macht Carnap allerdings selbst in der ungekürzten Autobiographie keine Angaben. Die einzige Quelle, die es dazu zu geben scheint, sind Carnaps Tagebücher aus dieser Zeit. Als Carnap und Gödel sich in den Jahren 1952 bis 1954 beide am Institute for Advanced Study in Princeton aufhalten, treffen sie sich alle paar Wochen für zwei bis drei Stunden. Während dieser Gespräche erörtern sie unter anderem Fragen der induktiven Logik, des Begriffs der Klasse sowie tagesaktuelle politische Themen und Alltagsfragen. Das geht aus Carnaps Tagebucheintragungen hervor, ${ }^{1}$ denn anders als in seinen Tagebüchern früherer Jahre referiert Carnap in diesem Zeitraum auch Argumente, hält aber nicht separat zusätzlich Gesprächsnotizen fest. Diese Quelle fehlt vorläufig.

Da die Tagebücher des genannten Zeitraums noch nicht transkribiert sind, lassen sich derzeit weder Gödels (zu seinen Lebzeiten nicht veröffentlichter) Aufsatz zu Carnap („Is mathematics syntax of language?“), an dem Gödel 1953 zu arbeiten begonnen hat, noch das Verhältnis der beiden Denker zueinander in dieser Periode neu betrachten. Gödels philosophische Notizbücher wiederum reichen zwar von 1934 bis 1955, Carnap wird dort aber, soweit man das derzeit beurteilen kann, in den Jahren 1946 bis 1955 nur noch selten namentlich erwähnt. Zum Einfluss Carnaps auf Gödel (Punkte II. und VI.) sowie zum Nutzen, den Carnap aus seinen Gesprächen mit Gödel in den gemeinsamen Wiener Jahren gezogen hat (Punkte III., IV. und V.), lässt sich hingegen bereits einiges festhalten.

\subsection{Carnaps Einfluss auf Gödel: Ein Fokus der Forschung}

Als wichtigen Einfluss für seine logischen Studien nennt Gödel selbst explizit im so genannten Grandjean-Fragebogen (einer Liste von Fragen, die ihm 1975 der Soziologe Burke D. Grandjean zugeschickt hat) neben der Lektüre von David Hilberts und Wilhelm Ackermanns Grundzüge der theoretischen Logik von 1928 den Besuch von Carnaps Vorlesung beziehungsweise seiner Übungen. ${ }^{2}$

\footnotetext{
${ }^{1}$ Diese Auskunft verdanke ich Dr. Brigitte Parakenings.

${ }^{2}$ Grandjean questionnaire, in: Kurt Gödel, CW IV, S 447. Im folgenden Textabschnitt wird erläutert, weshalb Gödel mit „lectures“ auch „Übungen“ gemeint haben könnte.
} 
Nach Warren Goldfarb hat Carnap Gödel in die Logik eingeführt. Goldfarb stützt sich dabei auf die Mutmaßung, Gödel habe die beiden Vorträge zur Logik gehört, die Carnap im Frühsommer 1928 bei den Treffen des Wiener Kreises gehalten hat. Vorgestellt wurde dabei Material aus dem Manuskript zu Carnaps Untersuchungen zur allgemeinen Axiomatik, in dem bereits die Themen Konsistenz, Vollständigkeit und Kategorizität eine wichtige Rolle spielen. Hingegen soll Schlicks Einführung in die Philosophie der Mathematik im Wintersemester 1925/26 Goldfarb zufolge keinen größeren Einfluss auf Gödel gehabt haben, denn Gödel habe danach zunächst keine weiteren Logikstudien betrieben.

Richtig ist, dass Gödel erst am 17. Oktober 1928 ein erstes logisches Werk zur selbstständigen Lektüre ausleiht; es handelt sich dabei um Gottlob Freges Grundlagen der Arithmetik. Im Wintersemester 1928/29 besucht Gödel laut seinem Studienbuch die philosophischen Übungen bei Carnap, welche dieser zu seiner Vorlesung „Die philosophischen Grundlagen der Arithmetik“ anbietet. ${ }^{3}$ Goldfarbs Einschätzung, Gödel sei von Carnaps Ausführungen weniger in mathematischtechnischer Hinsicht beeinflusst worden als in Hinsicht auf weiter zu klärende Grundlagenbegriffe von Axiomensystemen wie Konsistenz, Vollständigkeit oder logische Folgerung, ist Glauben zu schenken. ${ }^{4}$ In der Forschung wird plausibel nachgewiesen, dass Gödels Auseinandersetzung mit Carnaps Bemühungen um eine rein syntaktische Formulierung axiomatischer mathematischer Systeme zur Entstehung seines Unabhängigkeitstheorems im Jahr 1930 beigetragen hat. ${ }^{5}$

Das Lehrer-Schüler-Verhältnis zwischen Carnap und Gödel kehrt sich aber rasch nach ihrem Aufeinandertreffen um. Bereits einige Monate nachdem Gödel Carnaps Übungen zur Vorlesung „Die philosophischen Grundlagen der Arithmetik“ besucht hat, sucht Carnap in logischen Fragen regelmäßiger Gödels Rat, und sein Werk Logische Syntax der Sprache von 1934 ist auch eine Auseinandersetzung mit Gödels Unvollständigkeitstheorem. ${ }^{6}$ Das zeigt sich insbesondere in Carnaps Tagebüchern und seinen Gesprächsnotizen.

Eine weitere Etappe in der geistigen Auseinandersetzung zwischen Gödel und Carnap, die sich in Carnaps Tagebüchern und Gödels Philosophischen Notizbüchern widerspiegelt, ${ }^{7}$ betrifft den Begriff der Analytizität. Carnap versucht ihn als Kriterium für mathematische Wahrheit einzuführen, welche sich von der Beweisbarkeit in einem System unterscheidet. Die Notwendigkeit für eine solche Unterscheidung belegen Gödels Theoreme, die besagen, dass es in einem formalen System wahre Sätze gibt, die nicht beweisbar sind.

Im Großen und Ganzen zeigen Carnaps Tagebucheintragungen mit Bezug auf Gödel, die dazugehörigen Gesprächsnotizen sowie die sich anschließenden Briefe also, dass sich die inhaltliche Auseinandersetzung im Wesentlichen um Carnaps

\footnotetext{
${ }^{3}$ Vgl. Schimanovich (2002, S. 146) sowie Dawson (1999, S. 24) und Stadler (2015, S. 316).

${ }^{4}$ Vgl. auch Gödel (1986, S. 62, insb. Fn. 3).

${ }^{5}$ Awodey und Carus (2001, S. 154, 163 f.) und Goldfarb (2005, S. 188-190).

${ }^{6}$ Vgl. auch Goldfarb (2005, S. 192 f.).

${ }^{7}$ Vgl. dazu auch Awodey und Carus (2010, S. 263 f.).
} 
Manuskript der Logischen Syntax der Sprache gedreht hat, für das es Carnap sehr wichtig war, Gödels Urteil als Logiker einzuholen. So bleibt das Verhältnis maßgeblich als eines zur Klärung logischer Fragen definiert, die für Carnap selbst zwar von Anfang an auch philosophische Fragen sind, bei Gödel zu Beginn seiner Bekanntschaft mit Carnap im Binnenverhältnis zu ihm jedoch noch nicht vornehmlich philosophisch gedeutet werden. Nur so lässt sich die folgende Bemerkung Gödels erklären:

Max Phil IX, ${ }^{8}$ 18. November 1942-11. März 1943

[26] Bem[erkung]: Wieso eigentlich habe ich bis vor kurz[em] [bis Leibniz'] nicht einmal in der Logist [ik] die bedeutenden Autoren [nicht einmal Carnap] mit eigentlichem „Interesse an der Sache" gelesen [Beginn mit Herbrand ${ }^{10}$ ?]? ${ }^{11}$

Mit dieser Bemerkung kann nicht gemeint sein, dass Gödel Hilbert/Ackermann, Russell/Whitehead oder Frege nicht mit tatsächlichem Interesse gelesen habe. Da Gödel im Grandjean-Fragebogen angibt, er habe die Principia Mathematica von Alfred North Whitehead und Bertrand Russell bereits 1929 gelesen und der größte Einfluss auf seine Arbeiten von 1930 und 1931 sei die Lektüre von Hilbert/Ackermanns Werk gewesen sowie der Besuch von Carnaps Vorlesungen zur mathematischen Logik (1928/29), kann Gödel mit ,die Sache“ nicht Fragen der Logik als Teil- oder Grundlagendisziplin der Mathematik meinen. Gemeint ist vielmehr: Interesse an der Bedeutung der Logik für die Philosophie.

Der Hinweis Gödels, er habe „,bis vor kurzem“ Logik-Schriften nicht mit eigentlichem Interesse an der Sache gelesen, lässt sich zeitlich nicht treffsicher eingrenzen. Es liegt aber nahe anzunehmen, dass die Zeit nach dem intensiven Austausch zwischen Carnap und Gödel in den Jahren 1931/32 gemeint ist. Goldfarbs Analyse, Gödel sei durch Carnap zu seinen logischen Untersuchungen angeregt worden, ist daher in einem wesentlichen Punkt zu modifizieren: Gödels Interesse an der Philo-

\footnotetext{
${ }^{8}$ Diese und alle weiteren Transkriptionen aus dem Gödel-Nachlass sind von Eva-Maria Engelen neu angefertigt. Zum Teil standen dafür Vorversuche von Cheryl Dawson zur Verfügung. Das Material wird mit Erlaubnis des Institute for Advanced Study Princeton abgedruckt. Das Copyright an dem unveröffentlichten Material und alle Rechte daran verbleiben beim Institute for Advanced Study Princeton. (All works of Kurt Gödel used with permission. Unpublished material Copyright Institute for Advanced Study. All rights reserved by Institute for Advanced Study.) Die Transkriptionen der Carnap-Tagebücher sind von Brigitta Arden und Brigitte Parakenings, die Transkriptionen aus Carnaps Gesprächsnotizen stammen von Richard Nollan.

${ }^{9}$ Aus den Ausleihzetteln ergibt sich, dass Gödel am 18. Dezember 1929 Die philosophischen Schriften von Leibniz (d.i. der vierte Band der Gerhardt-Ausgabe) ausgeliehen hat. Die Bibliographie philosophischer Schriften, welche er sich in den Jahren 1936-1940 zusammengestellt hat, enthält außerdem Couturats Band zu Leibniz' Logik.

${ }^{10}$ Jacques Herbrand (1908-1931) war ein französischer Mathematiker. Gödel hat Arbeiten Herbrands 1931 gelesen, als dieser sie ihm zugeschickt hat. Herbrands Brief ist vom 7. April 1931, Gödels Antwort vom 25. Juli 1931. Vgl. Gödel (2003b, S. 14-20, 20-24).

${ }^{11}$ Die Vervollständigungen der Worte in eckigen Klammern stammen von E.-M. E., die Zusätze in den fett gedruckten eckigen Klammern sind von Gödel.
} 
sophie der Logik ist nicht vornehmlich durch Carnap, sondern durch Leibniz geweckt worden. ${ }^{12}$

\subsection{Gödel in Carnaps Tagebüchern von 1926 bis 1935}

Von Carnaps Tagebucheintragungen zwischen 1926 und $1935^{13}$ sind hier lediglich diejenigen ausgewählt, deren Aussagen weitergehende Rückschlüsse zulassen, als dass die beiden sich überhaupt getroffen haben.

Die Auswertung der Einträge ergibt, dass sich Carnap und Gödel im Jahr 1931 etwa vierzehnmal getroffen haben und 1932, als sich Carnap bereits vorwiegend in Prag aufhielt, immerhin noch viermal. Danach werden die Treffen seltener. Häufig waren die beiden dabei nicht alleine, sondern in einen größeren Kreis eingebunden, was zeigt, dass Gödel keineswegs ein separiertes Mitglied des Wiener Kreises gewesen ist, das lediglich schweigend an den Sitzungen zwischen 1926 und 1928 teilgenommen hat, wie man es seinen eigenen späteren Schilderungen nach vermuten könnte. Vielmehr war er, wie Carnaps Eintragungen zeigen, am regen philosophischen Austausch der Wiener-Kreis-Mitglieder beteiligt.

Das zeigt etwa der Eintrag vom 27. Januar 1930. Danach haben sich Gödel, Carnap, Friedrich Waismann, ${ }^{14}$ Carl Gustav Hempel, ${ }^{15}$ Rose Rand, ${ }^{16}$ Olga Hahn, ${ }^{17}$ Maria Kasper ${ }^{18}$ und Else Brunswik-Frenkel ${ }^{19}$ sowie Samuel Broadwin ${ }^{20}$ und Albert Blumberg ${ }^{21}$ abends im Café Reichsrat getroffen, um auf Anregung von Else Brunswik-Frenkel unter anderem ein Programm für Diskussionsabende zu planen. Zu bedenken ist in diesem Zusammenhang auch, dass Gödel sich daneben häufiger mit

\footnotetext{
${ }^{12}$ Awodey und Carus (2010) verweisen darauf, dass sowohl Carnap als auch Gödel Leibniz nachgeeifert haben und Carnaps Projekt einer Universalsprache durch Leibniz inspiriert war - ein Projekt, das mit Gödels Kritik an Carnaps Begriff des Analytischen gescheitert ist (vgl. Awodey und Carus 2010,S. 253, 262, 265, 270).

${ }^{13}$ Verwendet wurde der transkribierte Tagebuchtext ohne Kommentierung (Stand 5. Januar 2017), den Christian Damböck freundlicherweise zur Verfügung gestellt hat.

${ }^{14}$ Friedrich Waismann (1896-1959) ist ein österreichischer Philosoph, der Mitglied des Wiener Kreises war.

${ }^{15}$ Carl Gustav Hempel (1905-1997) ist ein deutscher Philosoph, der Mitglied des Wiener Kreises war.

${ }^{16}$ Die Philosophin Rose Rand (1903-1980) war Mitglied des Wiener Kreises.

${ }^{17}$ Die Mathematikerin Olga Hahn (1882-1931) war Mitglied des Wiener Kreises.

${ }^{18}$ Maria Kasper (um 1905-1989) war Mitglied des Wiener Kreises und ab 1931 mit Herbert Feigl verheiratet.

${ }^{19}$ Else Brunswik-Frenkel (1908-1958) ist eine Sozialpsychologin und war in Wien von 1931 bis 1938 Mitarbeiterin von Karl Bühler.

${ }^{20}$ Samuel Broadwin (1910-2008) war als Gastwissenschaftler von der John Hopkins University dabei.

${ }^{21}$ Albert Blumberg (1906-1997) ist ein amerikanischer Philosoph, der während seines Studienaufenthalts in Wien im Umfeld des Wiener Kreises anzutreffen. Er ist ein Koautor von Herbert Feigl.
} 
Herbert Feigl ${ }^{22}$ und Marcel Natkin ${ }^{23}$ in Wiener Caféhäusern zusammengefunden hat, etwa zum Diskutieren des Geist-Körper-Problems.

Ein weiterer Eintrag, der Gödels Beteiligung an den Treffen des Wiener-Kreises belegt, ist beispielsweise der vom 16. März 1930. Dort berichtet Carnap, dass er sich mit Neurath, ${ }^{24}$ Hempel, Feigl und Gödel getroffen habe und man über Atomsätze diskutiert habe. Die Auffassungen dazu sind offensichtlich unterschiedlich, denn während Neurath ,die Sprache möglichst erst dicht vor physikalischer Sprache beginnen lassen“ möchte, will sie Gödel ,vielleicht erst in der physikalischen Sprache“ anfangen lassen. Als Alternative überlegen sowohl Neurath als auch Gödel, ob man nicht mit der gewöhnlichen Alltagssprache beginnen sollte. Sie sind sich jedenfalls einig, dass sie nicht mit den Einzelempfindungen anfangen soll.

Offensichtlich war Gödel auch dabei, wenn erkenntnistheoretische Probleme besprochen wurden. Das legt ein Eintrag Carnaps vom 12. September 1930 nahe, dem zufolge sich unter anderem Carnap, Feigl, Gödel, Reichenbach, Dubislav, ${ }^{25}$ Grelling ${ }^{26}$ und Hempel im Café Dobrin getroffen haben und erkenntnistheoretische Fragen diskutiert haben.

Gödel interpretiert daneben die philosophischen Grundüberzeugungen der Mitglieder des Wiener Kreises und setzt sie in ein Verhältnis zueinander. Mit seinen Deutungen hält er Carnap gegenüber auch nicht hinter dem Berg, wie man im Tagebucheintrag Carnaps zum 7. Februar 1931 nachlesen kann: „Gödel hier. Über seine Arbeit. Ich sage, dass sie doch schwer verständlich ist. Meine Pläne eines Sprachaufbaus. Ich sage, dass ich alle Diskussionen im Zirkel usw. nur in dem Sinne als sinnvoll nehme, als sie Vorbereitungen für einen Sprachaufbau sind; er fürchtet, Waismann sei nicht dieser Ansicht; ich sei dann der einzige Positivist im Zirkel.“

Man kann Carnaps Tagebucheintragungen darüber hinaus entnehmen, welche Themen zwischen Carnap und Gödel intensiv diskutiert wurden. Dazu gehören insbesondere die Formalisierbarkeit der Mathematik, Gödels Entdeckung seines ersten Unvollständigkeitstheorems, die verzweigte Typentheorie (vgl. Carnaps Eintrag vom 4. März 1930) sowie Russells Antinomie (vgl. Carnaps Einträge vom 29. August 1930 und vom 17. September 1930), Carnaps damalige Aufsatz- und Buchprojekte sowie Gödels Pläne für akademische Arbeiten und sein berufliches Weiterkommen. Daneben erfährt man, inwieweit Gödel auch im Austausch mit einigen Mitgliedern des so genannten Berliner Kreises stand.

Hinsichtlich der Formalisierbarkeit der Mathematik sind insbesondere Carnaps Eintragungen vom 24. Januar, 8. Februar und 16. Februar 1930 aufschlussreich.

\footnotetext{
${ }^{22}$ Herbert Feigl (1902-1988) ist ein österreichisch-amerikanischer Philosoph, der im Wiener Kreis aktiv war.

${ }^{23}$ Marcel Natkin (1904-1963) war Philosoph und Fotograf, der bei Moritz Schlick promoviert hat. ${ }^{24}$ Otto Neurath (1882-1945), österreichischer Nationalökonom, Philosoph und Mitglied des Wiener Kreises.

${ }^{25}$ Walter Dubislav (1895-1937), deutscher Logiker und Wissenschaftstheoretiker, gemeinsam mit Hans Reichenbach und Kurt Grelling Begründer des Berliner Kreises.

${ }^{26}$ Kurt Grelling (1886-1942), deutscher Logiker und Wissenschaftstheoretiker; veröffentlichte 1936 einen Aufsatz über Gödels Unvollständigkeitstheorem.
} 
Carnap bezeichnet dort die Formalisierbarkeit als Gödels Problem und schlägt vor, die nicht formalisierbaren Fragen, Sätze und Begriffe als nicht eigentlich mathematische anzusehen.

Die Erwähnung der Entdeckung von Gödels erstem Unvollständigkeitstheorem fehlt in Carnaps Tagebuch selbstverständlich ebenfalls nicht. Der Ort, an welchem Gödel zuerst in sehr kleiner Runde (anwesend waren u. a. Carnap, Feigl und Waismann) von seiner Entdeckung berichtet hat, war das Café Reichsrat in Wien. Das lässt sich Carnaps Eintragungen vom 26. und 29. August 1930 entnehmen.

Am 6. September 1930, dem Tag, an dem Gödels Vortrag mit dem Titel „Über die Vollständigkeit des Logikkalküls" von 16:00-16:20 Uhr angesetzt war, lautet der entsprechende Eintrag in Carnaps Tagebuch dann:

Sa 6l Vorträge Tornier ${ }^{27}$ und Scholz ${ }^{28}$; dann Reichenbach ${ }^{29}$ „Physikalischer Wahrheitsbegriff“, Heisenberg ${ }^{30}$ „Kausalität und Quantenmechanik“; Diskussion. Nachmittags Vortrag Gödel „Vollständigkeit des Logikkalküls“; [...].

Wenn Carnap und Gödel sich alleine trafen, waren allerdings Carnaps Projekte sehr viel häufiger Gesprächsgegenstand zwischen den beiden als Gödels. So hält Carnap am 4. März 1930 fest, sie hätten sein Manuskript zum Logizismus diskutiert. Es dürfte sich dabei um das Manuskript handeln, welches nicht viel später als Aufsatz unter dem Titel „Die logizistische Grundlegung der Mathematik“ in der Zeitschrift Erkenntnis erschienen ist (Carnap 1931/32). Für Carnaps Arbeit dürften insbesondere die Treffen mit Gödel im Jahr 1931 und auch die weitaus weniger häufigen im Jahre 1932 wichtig gewesen sein. So notiert er am 7. Februar 1931, dass sie über seine Pläne eines Sprachaufbaus diskutiert hätten, am 21. April 1931 über Carnaps Entwurf zur Arithmetik, reelle Zahlen und Carnaps Versuch einer Logik ohne Existenzannahme. Am 10. Juni 1931 und am 12. Juli 1931 ist Carnaps Buchmanuskript Metalogik Thema zwischen den beiden. „Metalogik“ ist der Arbeitstitel für die ersten Entwürfe zu Carnaps Logische Syntax der Sprache, von denen außer dem Inhaltsverzeichnis nichts erhalten geblieben ist.

In diesem Manuskript beschäftigt sich Carnap unter anderem mit dem Begriff der Analytizität, ${ }^{31}$ welcher zudem bei den Treffen am 28. Juni 1931, am 12. Juli 1931 sowie in einem Brief von Gödel an Carnap vom 11. September 1932 zur Sprache kommt, den Carnap am 14. September 1932 in seinem Tagebuch erwähnt. Der Brief (Gödel 2003a, S. 346) enthält Gödels Kritik an Carnaps Definition des Begriffs , analytisch': Nach Gödel ist Carnaps Definition zirkulär, weil es keine Be-

\footnotetext{
${ }^{27}$ Erhard Tornier (1894-1982), deutscher Mathematiker. Ein Vortrag von Tornier ist im Programm der Tagung in Königsberg nicht genannt.

${ }^{28}$ Arnold Scholz (1904-1942), deutscher Mathematiker. Sein Vortrag bei der Tagung in Königsberg hatte den Titel „Über den Gebrauch des Begriffs Gesamtheit in der Axiomatik“.

${ }^{29}$ Hans Reichenbach (1891-1953), deutscher Philosoph und Physiker.

${ }^{30}$ Werner Heisenberg (1901-1976), deutscher Physiker.

${ }^{31}$ Vgl. Awodey und Carus (2010, S. 263). Zum Begriff der Metalogik bei Carnap vgl. Goldfarb (2005, S. 186). Da alle Begriffe innerhalb ein und desselben Systems definiert werden sollten, handelt es sich bei Carnaps Metalogik nicht um Metalogik in unserem heutigen Sinne.
} 
schränkungen für die Substituierbarkeit gibt, so dass unter den substituierten „Instanzen“" auch das Prädikat, das als analytisch definiert werden soll, sein könnte. Werden die Substituierungsmöglichkeiten aber eingeschränkt, gilt die Definition nur für Systeme, die für die klassische Mathematik nicht adäquat sind. ${ }^{32}$ Als sich Carnap und Gödel am 13. Dezember 1932 noch einmal im Café Museum treffen, ist die Definition von , analytisch", neben der von ,wahr', wieder ein Thema.

Aufschlussreich ist allerdings insbesondere Carnaps Eintrag vom folgenden Tag, dem 14. Dezember 1932, weil er zeigt, welchen Einfluss die Diskussionen mit Gödel auf Carnaps Denken gehabt haben, auch wenn Letzterer sich dessen nicht immer bewusst gewesen zu sein scheint:

Mi 14l [...] 1/2 7 Vortrag Menger „Die kr neue Logik“ in der Vortragsreihe „Die Krise der exakten Wissenschaften“. Gut, aber zu schwierig, zu viele unerläuterte Ausdrücke. - Wir fahren im Auto zum Museum; unter Neiders $^{33}$ Leitung tagt Neuraths Zirkel zur Physikalisierung der Psychoanalyse. Ich sage: Nicht einfach übersetzen, sondern Definitionen aufstellen, ferner Hypothesen mit hypothetischen Begriffen, mit Ableitungsregeln. Analog zu den Feldbegriffen. „Ich“ und „es“ nicht als Klasse von Vorgängen, sondern als Gebietsgröße. Neider sagt, dass sie sehr überrascht sind über die neue Auffassung, was mich wundert. Später sagt Gödel mir, dass ich diese Auffassung zum Teil auf seine frühere Anregung hin hätte (aber auch: Popper, Bernays, usw.). Es werden Zweifel geäußert, ob Neurath mit diesen Auffassungen einverstanden. (8.-10.) Dabei noch: Hollitscher $^{34}$ (aus Prag), Frau Ja-

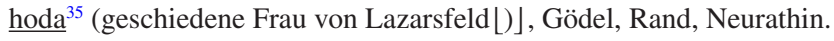

Dieser Eintrag Carnaps korrespondiert mit einer Bemerkung aus Gödels Philosophischen Notizbüchern Max 0 Phil I, die er im Jahre 1934 zu schreiben begonnen hat. Bei Gödel heißt es auf Manuskriptseite 72:

Bem[erkung]: Psychol[ogisch] versteht man die Begriffe mittels der „Typen“. Das sind spezielle (vollkommen spezialisierte) Begriffe, welche gewissermaßen in der Mitte des Gebietes jedes Begriffs liegen. Die nicht typischen Exemplare sind gewissermaßen eine Mischung zwischen dem Typus und seinem Gegenteil (oder konträren Typ) - am deutlichsten bei einer Farbe. Die Erkenntnis mittels des Typus und dem Grad \{der\} Abweichung ist exakter (das ist vielleicht ein heuristisches mat[hematisches] Prinzip. Die Aussage: , $x$ ist typisch gelb“ gibt gewissermaßen eine vollständige Information).

Nimmt man Carnaps Eintrag zwei Jahre zuvor am 14. Dezember 1932 für bare Münze, dann hat er den Gedanken der Gebietsgröße von Gödel übernommen. ${ }^{36}$ Das zeigt, dass sich die beiden auch über philosophische Fragen ausgetauscht haben, ${ }^{37}$

\footnotetext{
${ }^{32}$ Vgl. auch Goldfarb (2003, S. 338) sowie Awodey und Carus (2010, S. 264).

${ }^{33}$ Heinrich Neider (1907-1990), Doktorand von Moritz Schlick.

${ }^{34}$ Walter Hollitscher (1911-1986), österreichischer Philosoph.

${ }^{35}$ Marie Jahoda (1907-2001), österreichische Psychologin.

${ }^{36}$ „Analog zu den Feldbegriffen. ,Ich“ und ,es“ nicht als Klasse von Vorgängen, sondern als Gebietsgröße. Neider sagt, dass sie sehr überrascht sind über die neue Auffassung, was mich wundert. Später sagt Gödel mir, dass ich diese Auffassung zum Teil auf seine frühere Anregung hin hätte." Siehe unten Carnaps Eintrag vom 14. Dezember 1932.

${ }^{37}$ Aus dem Tagebucheintrag vom 7. Februar 1931 geht zwar hervor, dass Carnaps philosophisches Projekt „Sprachaufbau“ Gegenstand der Unterhaltung gewesen ist, Gödels Gesprächsbeitrag wird jedoch als auf dessen eigene Arbeiten eingeschränkt geschildert.
} 
die über das engere Gebiet der Logik hinausgehen. Darüber hinaus wird deutlich, dass Gödel eigene Auffassungen dargelegt hat und diese drittens bei Carnap immerhin so sehr Wirkung gezeigt haben, dass er sie in sein eigenes Denken einbezogen hat, sich dessen aber nicht mehr bewusst war.

Carnap hat bekanntermaßen häufiger ihm einleuchtende Gedanken anderer Philosophen ohne Namensnennung aufgenommen und in seine eigenen Überlegungen integriert. Aus dem Umstand allein, dass das auch in Gödels Fall so gewesen zu sein scheint, ist daher noch nicht abzulesen, dass Carnap Gödel nicht als umfassenden philosophischen Denker wahrgenommen hat. Diese Einschätzung ergibt sich vielmehr aus dem Gesamtbild, zu dem insbesondere Carnaps Gesprächsnotizen beitragen. Aus ihnen lässt sich unten in Abschnitt IV des vorliegenden Beitrages noch genauer ablesen, welche Rolle Gödel für Carnap gespielt hat.

Jedoch werden Erörterungen von Gödels Arbeit in Carnaps Tagebuchaufzeichnungen durchaus erwähnt, allerdings sehr viel spärlicher. So heißt es am 23. Dezember 1930:

Di 23। [...] 1/2 3 - 5 mit Gödel. Über seine Metamathematik (Korrekturbogen); sehr interessant.

Da Ende 1930 die Rede von einem Korrekturbogen ist, kann es sich entweder um Gödels Aufsatz „Über formal unentscheidbare Sätze der Principia Mathematica und verwandter Systeme I" handeln, der 1931 in Band 38 der Monatshefte für Mathematik und Physik erschienen ist (Gödel 1986, S. 144-194), oder, unwahrscheinlicher, um den Beitrag „Diskussion zur Grundlegung der Mathematik“, der im ersten Heft des zweiten Bandes von Erkenntnis im Jahre 1931 erschienen ist (Gödel 1986, S. 200-204). Carnap nennt Gödels Ergebnisse im zitierten Eintrag zwar ,sehr interessant“", scheint aber Schwierigkeiten gehabt zu haben, Gödel zu folgen, wie sich aus seinem Eintrag vom 7. Februar 1931 ergibt, wo er bemerkt, er habe mit Gödel über seine Arbeit gesprochen, fände sie jedoch ,,doch schwer verständlich“.

Einige Eintragungen Carnaps in Bezug auf Gödels Äußerungen verbleiben leider Andeutungen. So heißt es am 15. Dezember 1932: „Gödel; er sagt, die inhaltliche Redeweise sei nicht unzulässig." Leider wird nicht ausgeführt, wie Gödel das inhaltlich begründet. Ebenso wenig wie die Bemerkung vom 7. Juli 1932, nach welcher Gödel „,vom Zirkel sehr unbefriedigt“ sei. Man könnte dies auf die in demselben Eintrag erwähnte Haltung von Waismann und Schlick beziehen, die aber auch nicht weiter beschrieben wird. Schade ist das insbesondere, weil wir so nicht erfahren, welche philosophischen Motive Gödel vom Wiener Kreis sehr unbefriedigt sein lassen. Wir wissen darüber zwar einiges aus Gödels Sicht; diese ist allerdings retrospektiv mit einigen Jahren Abstand zu den Ereignissen formuliert.

Zudem erfahren wir über Gödels berufliche Pläne bei Carnap einiges, was mit Gödels eigenen Äußerungen korrespondiert. So heißt es am 7. Juli 1933: „Gödel hier. Er ist für $1 \mathrm{Jahr}$ zum Flexner Institut nach Princeton berufen, durch Veblen ${ }^{38}$ und von Neumann, ${ }^{39}$ für Grundlagen der Mathematik. Vielleicht will er drüben blei-

\footnotetext{
${ }^{38}$ Oswald Veblen (1880-1960), US-amerikanischer Mathematiker.

${ }^{39}$ John von Neumann (1903-1957), ungarisch-amerikanischer Mathematiker.
} 
ben, wenn's in Europa immer schlimmer wird. Allerhand über Politik." Spätere Eintragungen Carnaps über Gödels berufliches Weiterkommen sind dann vor allen Dingen solche aus zweiter Hand, wie die Notiz vom 30. Dezember 1935 zeigt:

Symposium über Wahrscheinlichkeit: Morris Cohen, Northrop, Savery. ${ }^{40}[\ldots]$ Dazu kommt Irving (Princeton), ${ }^{41}$ der mir hilft, die Beziehungen zur amerikanischen Philosophie anzugeben. [...] - (Er sagt, dass Gödel kürzlich nach Wien zurück ist, nervöser Zusammenbruch; Veblen habe gesagt: zu viel Introspektion.) - [...].

Zu Gödels Kontakten mit einigen Mitgliedern des so genannten Berliner Kreises erfahren wir in Carnaps Tagebüchern einiges, z. B. dass er am 12. September 1930 dabei war, als bei einem Treffen im Café Dobrin neben Feigl, Gödel, Hempel und Reichenbach Dubislav ${ }^{42}$ und Grelling ${ }^{43}$ anwesend waren. Dubislav findet im Zusammenhang mit Gödel zudem am 28. Februar 1931 sowie am 17. März 1931 Erwähnung.

\subsection{Gödel in Carnaps Gesprächsnotizen von 1928 bis 1953}

Wertet man Carnaps von 1928 bis 1953 reichende Gesprächsnotizen aus, lässt sich Carnaps spezifisches Interesse an Gödel leicht erkennen: Er konsultiert ihn als mathematischen Logiker, nicht als philosophisch arbeitenden Denker, obgleich sie, wie die Tagebuchaufzeichnungen zeigen, nicht nur über Fragen der Logik diskutiert haben. Carnap scheint gehofft zu haben, Gödel könne ihm bei den Problemen, eine logische Syntax der Sprache zu verfassen, helfen; darauf weisen die fünf Eintragungen „Gödel fragen“ hin, die allesamt aus dem Jahr 1931 stammen.

Im Folgenden sei der Inhalt der Gesprächsnotizen jeweils kurz angegeben; es handelt sich nicht um eine wörtliche Wiedergabe des Notierten. Wörtliche Zitate stehen in Anführungszeichen. ${ }^{44}$

\section{Gesprächsnotizen ${ }^{45}$}

(RC 102-43-09) 14.12.1928 Gesprächsthema: Verhältnis von formaler Sprache und

Physik; Logik und Mathematik als rein formale Verfahren.

(RC 081-01-32) 14.12.1928 Gödel zur Axiomatik

\footnotetext{
${ }^{40}$ Vgl. Philosophical Review 45 (1936), S. 173, wo das Symposium „Implications for Philosophy of the Theory of Probability" von Morris R. Cohen, F. S. C. Northrop und William Savery annonciert ist. Morris Raphael Cohen (1880-1947), F. S. C. Northrop (1893-1992) und William Savery (1875-1945) waren amerikanische Philosophen.

${ }^{41}$ John Allan Irving (1903-1965), kanadischer Philosoph, der zunächst in Princeton gelehrt hat.

${ }^{42}$ Siehe Fußnote 25.

${ }^{43}$ Siehe Fußnote 26.

${ }^{44}$ Die Rechtschreibung der Transkription wurde stillschweigend angepasst.

${ }^{45}$ Die Transkriptionen der Carnapschen Gesprächsnotizen von Richard Nollan werden vor der Veröffentlichung überarbeitet werden. Da sie zum textuellen Kontext der Tagebuchnotizen gehören, plant Christian Damböck, sie in die Edition von Carnaps Tagebüchern aufzunehmen.
} 
(RC 102-43-22) 23.12.1929 Gespräch mit Gödel über Unerschöpflichkeit der Mathematik und den Umstand, dass es keine Characteristica universalis und kein Entscheidungsverfahren für die gesamte Mathematik geben kann.

Der Notiz ist nicht zu entnehmen, von wem die jeweiligen Aussagen oder Schlussfolgerungen stammen. Interessant ist, dass im Zusammenhang mit der Unerschöpflichkeit der Mathematik immer wieder von dem sich daraus ergebenden Erfordernis der „Anschauung“ die Rede ist.

(RC 102-43-21) 14.03.1931 „Gödel fragen!““ Carnap listet fünf Fragen auf, die er mit Gödel diskutieren will. Fragen 1 und 3 betreffen Ausführungen von Hilbert, Frage 2 ist eine nach der Eliminierung von Identität, Frage 4 betrifft den Gebrauch einer Regel an Stelle eines Axioms, Frage 5 die Werttafel von Dubislav.

(RC 102-43-20) 19.03.1931 Diskussion von Carnaps Regeln 1a und 1b. Carnap notiert, was Gödel im Einzelnen dazu meint.

(RC 102-43-19) 21.04.1931 Sehr kurze Gesprächsnotiz zum Thema Grundlegung der Arithmetik.

(RC 102-43-18) 09.06.1931 „Gödel fragen: 1.) Ich möchte ohne Satzvariable, Prädikatsvariable (und Zahlenfunktionsvariable) auskommen. Bedenken? Dagegen wohl Zahlvariable (auch als freie Variable, aber nur bei logischer Allgemeinheit).

Gödel: Dies ist gut; da wird Existenz (unbeschränkt) nicht ausdrückbar.“

Danach folgen insgesamt noch drei Fragen: eine zur Gleichungsregel, eine zu Prädikaten und Zahlfunktionen in der Arithmetik und die dritte und letzte zum Begriff der Beweisbarkeit. Mit Ausnahme der zweiten Frage sind die Positionen von Gödel und Carnap dazu jeweils notiert.

(RC 102-43-17) 10.06.1931 Gespräch mit Gödel über Finitismus. ${ }^{46}$

(RC 102-43-15) 02.07.1931 Im Anschluss an Notizen zu Ausführungen von Gödel während einer Sitzung des Wiener Kreises listet Carnap zwei Fragen an Gödel auf. Die erste Frage lautet: ,Was ist mit „,höherem Schema“ gemeint? [...] Kann man nicht ein allgemeines Schema für rekursive Definitionen aufstellen?" Die zweite Frage betrifft die Entscheidbarkeit konkreter Sätze in einer Sprache.

(RC 102-43-14) 12.07.1931 Drei Fragen zu Gödels Mathematik und Metamathematik sowie Gödels Antworten dazu.

(RC 102-43-13) Keine Gesprächsnotiz, sondern eine Notiz zu Zermelos Brief vom 21. September 1931 an Gödel.

(RC 102-43-16) 26.01.1932 Bei dieser Notiz handelt es sich nicht um eine Gesprächsnotiz, sondern um ein Herbrand-Exzerpt zu Gödel.

(RC 102-43-12) 24.03.1932 Diskussion über Carnaps Manuskript (Arbeitstitel „Metalogik ${ }^{647}$ ); rekursive Definition; , beweisbar', ,richtig '; Rolle von Naturgesetzen; ,Gehalt', etc.

\footnotetext{
${ }^{46}$ Vgl. dazu auch Carnaps Tagebucheintrag vom 8. Februar 1930.

${ }^{47}$ Das Manuskript erscheint später als Logische Syntax der Sprache (Carnap 1934).
} 
(RC 102-43-11) 08.12.1932 und 13.12.1932: 08.12.1932: Gödels Äußerungen zu rekursiver Definition. 13.12.1932: Carnaps Verfahren für unentscheidbare Sätze; deskriptive Antinomie; Funktionsvariablen und Prädikatvariablen. Frage nach der Zulässigkeit inhaltlicher Redeweise (Beispiel: Zahlen sind nicht Dinge). Formulierung eines Toleranzprinzips durch Gödel: „Wir tun gut daran, bei logischen Erörterungen die formale Redeweise anzuwenden.“

(RC 102-43-10) Juli 1933 Carnap diskutiert mit Gödel: a-Begriffe (ableitbar, beweisbar, widerlegbar, a-unentscheidbar), f-Begriffe (Folge, gültig, widergültig, unentscheidbar) und L-Begriffe (L-Folge, L-gültig = analytisch, L-widergültig = kontradiktorisch, L-unentscheidbar = synthetisch) sowie ,Synonymität"; Rolle von Naturgesetzen; u. a.

(RC 102-43-08) 29.06.1935 Definition von , analytisch ‘. ${ }^{48}$

(RC 102-43-06) 13.11.1940 Gödel über eine mögliche Theorie, die Begriffe wie ,Gott', ,Seele“ und ,Ideen“ in ihrem Postulatensystem enthält sowie Carnaps Einwände dagegen. ${ }^{49}$,Ich [= Carnap]: Ich denke aber, diese Richtungsänderung wäre sicher unfruchtbar. Zu dieser Annahme (die natürlich nicht Beweis ist) trägt bei, dass wir durch Psychoanalyse usw. wissen, wie die Gottesvorstellung und ganze Theologie usw. auf gewisse Kindheitserlebnisse und Vorstellungen zu-

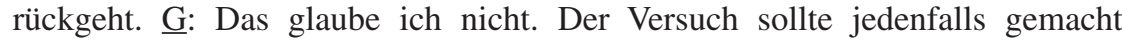
werden."

(RC 102-43-07) 14.11.1940 Diskussion zu Gödels Vortrag über die Kontinuumshypothese.

(RC 088-30-03) 26.03.1948 Die jüngste Gesprächsnotiz Carnaps über eine Unterhaltung mit Gödel scheint diejenige vom 26. März 1948 zu sein, als beide sich in Robert Oppenheimers Haus getroffen haben. Carnap berichtet über Gödels Leibniz-Studien und was sich Gödel hinsichtlich eines Entscheidungsverfahrens für die Mathematik davon verspricht. Es ist eines der wenigen Gesprächsprotokolle zu Gödel, in denen Carnap ausschließlich schildert, was Gödel denkt. Das Protokoll zeigt die innerliche Distanziertheit Carnaps von Gödels Darlegungen. Diese wird gleich zu Beginn offenbar, wenn er schreibt, Gödels Mutmaßung, einiges von Leibniz' unveröffentlichten Schriften werde absichtlich zurückgehalten, sei ein Ausdruck seiner Verfolgungsidee. Carnap benutzt nicht das Wort ,Verfolgungswahn“, meint das aber.

Inhaltlich ist diese Gesprächsnotiz in vielen Hinsichten von Interesse. Carnap beschreibt, wie Gödel Leibniz’ Idee eines allgemeinen Entscheidungsverfahrens für

\footnotetext{
${ }^{48}$ Carnap hat sich seit 1931 um eine Definition von, analytisch 'bemüht, die es ermöglichen sollte als Kriterium von Wahrheit zu fungieren, das sich von dem für Beweisbarkeit unterscheidet. Vgl. Awodey und Carus (2010, S. 263).

${ }^{49}$ Was Gödel hier vorschlägt, ist das, was er an anderer Stelle eine wissenschaftliche, exakte Theologie genannt hat. Punkt 13 seiner philosophischen Ansichten: „Es gibt eine wissenschaftliche (exakte) $P h[$ ilosophie $\}$ und Th[eologie $]$ und dies[e] ist auch für die Wissenschaften höchst fruchtbar.\}, welche die Begriffe der höchsten Abstraktheit behandelt“" (undatiertes Blatt aus Gödels Nachlass, ursprüngliche Dokumentennummer 060168).
} 
die Mathematik als eines konzipiert, das nicht durch eine Maschine durchführbar sei, weil es nicht spezifisch genug sein könne.

Carnap gibt in der Notiz nicht zu erkennen, wie er zu dem über die reine Logik hinausgehenden Inhalt des von Gödel Gesagten an dieser Stelle steht, und auch nicht, was er von Gödels Analogie zwischen theoretischer Physik und Mengenlehre hält. Er verweist nur darauf, dass sie sich hinsichtlich der Aufgabe von Hilberts Zielen einig seien, insofern sie diesen Umstand nicht, wie Tarski, ${ }^{50}$ als Grund zum Verzweifeln ansehen, und bringt mit einem knappen Satz lediglich seine Enttäuschung darüber zum Ausdruck, dass Gödel seine Arbeiten nicht zur Kenntnis genommen hat: „,̈̈ber induktive Logik: Er hat meine Aufsätze nicht gelesen.“

Insgesamt zeigen die Gesprächsnotizen deutlich, wie sehr sich Carnap in der Anfangszeit seiner Arbeit an dem Buch, das 1934 unter dem Titel Logische Syntax der Sprache erscheinen sollte, auf Gödels mathematisches Urteil verlassen hat. Man kann sogar darüber hinausgehen und sagen, dass er sich in wesentlichen Fragen Anregungen oder gar Lösungen von Gödel erhofft hat und die ein oder andere Hilfestellung von Gödel auch erhalten hat. ${ }^{51}$ Im Laufe des Jahres 1931 notiert Carnap mehrmals ausdrücklich: „Gödel fragen“ und listet dann eine Reihe von Fragen auf, die er Gödel gestellt hat. Philosophische Fragen, die über logische hinausgehen, kommen lediglich in drei der Gesprächsnotizen vor: Einmal in der vom 23. Dezember 1929, aus der kein Gesprächsverlauf erkennbar ist, und dann erst wieder in denen vom 13. November 1940 und 26. März 1948, aus deren Duktus Carnaps ablehnende Haltung gegenüber Gödels Äußerungen klar erkennbar ist.

\subsection{Briefwechsel zwischen Carnap und Gödel}

Nimmt man noch den Briefwechsel zwischen Carnap und Gödel hinzu, der überwiegend aus dem Jahr 1932 stammt, als Carnap in Prag weilte, sieht man, dass sich auch hier die Diskussion um die Themen für Carnaps Logische Syntax der Sprache dreht und um Gödels Einwände dazu.

Wie Warren Goldfarb in seiner Einleitung zur Carnap-Gödel-Korrespondenz bereits herausgearbeitet hat, ${ }^{52}$ ist der Anlass der Korrespondenz Carnaps Arbeit an dem Manuskript, welches er unter dem Titel Logische Syntax der Sprache (Carnap 1934) veröffentlichen sollte. Er wollte damit zeigen, dass mathematische und logische Wahrheiten keine Beschreibungen einer wie auch immer gearteten Realität seien, sondern reine Artefakte. Gödels Unvollständigkeitstheorem stellt für ein solches Vorhaben ein Problem dar, denn danach kann eine Sprache, die alle mathematischen Wahrheiten ergeben soll, nicht durch ein Axiomensystem und Ableitungsregeln bestimmt sein, weshalb Carnap eine Definition der Wahrheit nur für

\footnotetext{
${ }^{50}$ Alfred Tarski (1901-1983), polnischer Mathematiker.

${ }^{51}$ Vgl. Carus (2007, S. 251).

${ }^{52}$ Goldfarb (2003, bes. 337 f.).
} 
mathematische oder logische Sätze einer Sprache geben wollte und eine Definition von , analytisch“ für diese Sprache. Gödel macht Carnap darauf aufmerksam, dass seine Definition von , analytisch` (und daher auch der W-Beweis) fehlerhaft ist.

\subsection{Carnap in Gödels philosophischen Notizbüchern}

Die Erwähnungen Carnaps in Gödels philosophischen Notizbüchern zeigen, dass Gödel Diskussionen mit Carnap und seine Lektüre von Carnaps Schriften in seine eigenen philosophischen Überlegungen integriert hat. Ehe sich Gödel der Logik als einem philosophischen Gebiet gewidmet hat, war Carnaps Vorlesung für Gödels logisch-mathematische Arbeiten von 1930 und 1931 von Bedeutung, aber danach, als er durch seine Leibniz-Lektüre die Bedeutung der Logik für die Philosophie eruiert, greift er auf Carnaps Überlegungen und Erörterungen zurück und berücksichtigt dessen Überlegungen zur Logik in seinen eigenen philosophischen Reflexionen.

Gödel hat über einen Zeitraum von 22 Jahren (1934-1955) seine philosophischen Bemerkungen, die so genannten Maximen/Philosophie (Max Phil), die uns in 15 Notizbüchern überliefert sind, in der Kurzschrift Gabelsberger niedergeschrieben. Sie enthalten persönliche Leitfäden zur Lebensführung ebenso wie philosophische Lektürelisten, Zitate anderer Autoren, sowie eigene Gedanken und systematische philosophische Reflexionen. Die Zählung der Max Phil beginnt bei 0 und endet bei XV; Heft XIII ist Gödels eigenen Angaben zufolge verloren gegangen.

Da bei Weitem nicht alle Max-Phil-Hefte Gödels transkribiert sind, kann das, was sich derzeit zu Gödels Beschäftigung mit Carnaps Philosophie sagen lässt, nur unvollständig und vorläufig sein. Es lässt sich allerdings bereits festhalten, dass Carnap im ersten Notizheft (= Max 0) und im letzten (= Phil XV) Notizbuch genannt wird. Dazwischen taucht sein Name immer wieder einmal auf.

In Max 0 Phil I, also dem ersten Notizheft, in dem Gödel zwischen Oktober 1934 und Juni 1941 Notizen festgehalten hat, fällt Carnaps Name auf Manuskriptseite 3 in Zusammenhang mit einem Lektüreprogramm, das Gödel für sich aufstellt. Dieses Arbeits- und Lektüreprogramm beginnt mit Werken Karl Bühlers und Franz Brentanos, denen Heinrich Gomperz ${ }^{53}$ zweibändiges Werk zur Weltanschauungslehre folgt. Es wird ergänzt durch Studien in Überwegs Grundriß der Geschichte der Philosophie. Als weitere Lektüredesiderata notiert Gödel die Werke Georg Wilhelm Friedrich Hegels, das Studium von Bernard Bolzanos Wissenschaftslehre, das Studium der antiken Philosophen Aristoteles und Plato sowie das der Geschichte der modernen Philosophie bis 1900. Die Schriften der Autoren Karl Bühler, Max Scheler, Edmund Husserl und Franz Brentano, die stellvertretend für ein Studium der Psychologie und Phänomenologie stehen, sind gesondert angeführt. Dann fol-

\footnotetext{
${ }^{53}$ Gödel bezeichnet Gomperz im Grandjean-Fragebogen als seinen Lehrer. Gomperz hatte in Wien seinen eigenen Kreis, den so genannten Gomperz-Kreis, den auch Carnap besuchte.
} 
gen bekannte Namen der positivistischen Philosophie: An erster Stelle steht Rudolf Carnap, gefolgt von Alfred Whitehead, Bertrand Russell, Charles W. Morris sowie Moritz Schlick, Ludwig Wittgenstein und Friedrich Waismann.

Gödels Beschäftigung mit Carnaps Philosophie steht also bereits zu Beginn von Gödels philosophischen Arbeiten auf dem Programm. Dass er dieses Programm auch abgearbeitet hat, zeigen die erhaltenen Notizbücher, in denen die Beschäftigung mit den genannten Autoren ihren Niederschlag gefunden hat.

Diese Feststellungen sind geeignet, Gödels überlieferte Aussage, er sei zwar Mitglied des Wiener Kreises gewesen, habe sich philosophisch aber stets in Opposition zu diesem befunden, in einem entscheidenden Punkt zu präzisieren: Gödel war in Opposition zur Philosophie des Wiener Kreises, insbesondere zu der des so genannten linken Flügels, zu dem Carnap zu zählen ist, hat aber seine eigenen Auffassungen auch in Auseinandersetzung mit den im Wiener Kreis vertretenen Positionen und ihren Vertretern, also insbesondere Carnap, entwickelt. Das lässt sich nicht zuletzt daran ablesen, dass Gödel sich mit Carnaps Philosophie zwischen 1934 und 1955 befasst hat, und zwar nicht nur mittels passiver Lektüre.

So findet sich in Heft Zeiteinteilung Max II zwischen 1938 und 1940 auf Manuskriptseite 148 der Eintrag: „Carnap und Veblen schreiben.“ Der Eintrag ist durchgestrichen, was darauf hindeutet, dass Gödel es sich nicht nur vorgenommen, sondern beiden tatsächlich geschrieben hat. Allerdings ist aus diesem Zeitraum kein Brief Gödels an Carnap erhalten. Ein Gutteil des Briefwechsels zwischen Gödel und Carnap ist in Band IV von Gödels Collected Works (Gödel 2003a) ediert und publiziert. Die überwiegende Zahl der Briefe wurde 1932 geschrieben, danach folgen nur noch in den Jahren 1933, 1934, 1935 und 1939 je ein Brief mit inhaltlichen Bezügen von Carnap an Gödel. Daraus könnte man schließen, dass Gödel selbst sich nach 1932 nicht mehr mit Carnaps Ideen befasst hat. So ist es aber nicht, was Gödels Philosophische Notizbücher zeigen. Interessant ist, wie sich Gödel in seinen Philosophischen Notizbüchern mit Carnap als Philosoph auseinandersetzt, und dass Diskussionen mit Carnap in Gödels philosophischen Überlegungen noch zehn Jahre, nachdem sie geführt wurden, eine Rolle spielen.

Schauen wir uns dafür einige von Gödels Bemerkungen an, in denen er auf Carnap zu sprechen kommt. Inhaltlich können sie im Rahmen dieses Aufsatzes nicht interpretiert werden, da das den vorliegenden Rahmen sprengen würde. Hier kommt es auf Folgendes an: (1) Die Ausschnitte legen nahe, dass Gödel sich der Carnapschen Philosophie erst nach den Diskussionen im Rahmen von Carnaps Arbeit an Logische Syntax der Sprache in vollem Umfang zugewendet hat, sie dann aber sorgfältig rezipiert hat.

Für diese These spricht die bereits oben interpretierte Bemerkung aus Heft Max Phil IX, Manuskriptseite 26:

Bem[erkung]: Wieso eigentlich habe ich bis vor kurz[em] [bis Leibniz] nicht einmal in der Logist $[i k]$ die bedeutenden Autoren [nicht einmal Carnap] mit eigentlichem ,Interesse an der Sache' gelesen [Beginn mit Herbrand?]?

Gödel ist selbst darüber erstaunt, Carnap mit seinen Überlegungen zur Logik nicht von Anfang an immer auch in philosophischer Hinsicht verstanden und gedeutet zu haben. 
Weitere Bemerkungen aus Heft Max Phil IX, in denen Carnaps Überlegungen eine Rolle spielen, sind die folgenden:

Max Phil IX, 18. November 1942-11. März 1943

[29] Bem[erkung] (Phil[osophie]): Merkwürdiger Weise nimmt man die Objekte, welche man eigentlich durch theoretische Konstruktion erreichen kann [fremdpsychische Vorgänge, ${ }^{54}$ Objekte der Außenwelt], genauso ,unmittelbar“ wahr wie die eigenen Sinneswahrnehmungen. Das kann zwei Gründe haben: 1. Man nimmt stattdessen einen „Ersatz“wahr, den man [fälschlich] damit ,,identifiziert“, ähnlich wie man Symbol und Bezeichnetes identifiziert. 2. Das Gegebene [die Sinnesempfindungen] dient nur dazu, die Aufmerksamkeit auf ein unmittelbar wahrnehmbares Objekt zu richten, und man könnte dies auch ohne Sinnesempfindung wahrnehmen, wenn man wüsste, wie man die Aufmerksamkeit zu dirigieren hat [das bedeutet die Möglichkeit der Gedankenübertragung]. Sicher kann man in einem gewissen Sinn die empirischen Dinge ohne Empfindungen wahrnehmen, indem man sie aus der Theorie der Welt konstruiert.

In dieser Bemerkung verwendet Gödel mit dem Begriff ,fremdpsychische Vorgänge “ einen Terminus aus Carnaps Der logische Aufbau der Welt, was dafür spricht, dass sich die Beschäftigung mit Carnaps Philosophie bei Gödel tief eingeprägt hat. Zwar könnte Gödel den Ausdruck auch von Max Scheler übernommen haben; aber die Weise, wie Gödel hier darüber nachdenkt, inwiefern fremdpsychische Vorgänge „,unmittelbar“" wahrnehmbar sind (nicht etwa ,,analog“, also abgeleitet), ist den Positionen des Wiener Kreises geradezu entgegengesetzt, so dass von einer indirekten Bezugnahme auf Carnap auszugehen ist.

Max Phil IX, 18. November 1942-11. März 1943

[35] Bem[erkung] (Phil[osophie]): Die Deflinition] der Wahrheit ist richtig in der Transzend[enten] Theorie, aber es muss [ein] immanentes Wahrheitskriterium geben, und diesem gegenüber muss dann die Transc[endente] Def[inition] als eine „nutzlose Tautologie“ erscheinen [ähnlich wie Carnaps Def[inition] von ,,analytisch“].

Wir haben bereits gesehen, wie ausführlich Gödel und Carnap den Begriff , analytisch ' diskutiert haben und welche Bedeutung das Konzept für Carnaps Logische Syntax der Sprache hatte. Gödel kritisiert Carnaps Definition hier als nutzlos. Selbst diese Bezugnahme zeigt aber, wie sehr bei Gödel die Diskussionen mit Carnap vom Beginn der 30er-Jahre des 20. Jahrhunderts nachhallen.

Max Phil IX, 18. November 1942-11. März 1943

Bem[erkung] (Phil[osophie]): Der Unterschied zwischen „leer laufender“ und inhaltsreicher Theorie ist:

1. Im ersten Fall [sind] die Dinge konstituierbar eines nach dem anderen; im zweiten Fall nur simultan.

2. Im ersten Fall [ist] jede Aussage, welche die neue[n] Dinge enthält, übersetzbar in eine ohne diese; im zweiten Fall nur gewisse. Dagegen gibt es viele (für Theorien wichtige) [Aussagen], die nur insofern etwas sagen, als die Möglichkeit der eingeführten Dinge mit den eingeführten Eigenschaften behauptet wird. Dann muss man aber zum Sinn einer solchen Aussage immer die ganz[en] Axiome, durch die Dinge impl[izit] definiert werden, dazurechnen (andernfalls würden einander widersprechende Aussagen wahr sein). Aber selbst wenn man sie dazurechnet,

\footnotetext{
${ }^{54}$ Vgl. etwa Carnap (1928, Abschn. IV, C): „Die obersten Stufen: Fremdpsychische und geistige Gegenstände.“
} 
kann dasselbe eintreten [wegen unentscheidbaren Sätzen, und eine vollständige Charakterisierung ist eine reine [74] Chimäre]; außerdem, selbst die Chimäre angenommen, würden dann alle Folgerungen aus den $A x[i o m e n]$ dasselbe besagen. Wenn man die Entscheidbarkeit im objektiven Sinn (nach Carnap) herstellen will, so braucht man dazu den Klassenbegriff. Insofern ist vielleicht der Klassenbegriff allein schon für alle solche „Schöpfungen“ hinreichend? Aber es kann sein, [dass] man viel[leicht] etwas offen lässt (oder muss es).

Carnap hat sich in Logische Syntax der Sprache mit Entscheidbarkeit und Unentscheidbarkeit auseinandergesetzt: , $S_{I}$ heißt entscheidbar, wenn $S_{l}$ beweisbar oder widerlegbar ist; andernfalls unentscheidbar" (Carnap 1934, S. 85). Hier gilt daher desgleichen, was bezüglich der vorangegangenen Bemerkung festgestellt wurde: Für Gödel ist selbst zehn Jahre nach seinen intensiven Diskussionen mit Carnap dieser noch ein wichtiger, wenn auch räumlich abwesender, Gesprächspartner, auf dessen einmal gemachte Äußerungen sich Gödels inhaltliche Gedanken nach wie vor beziehen.

\section{Max Phil X, 1943-1944}

[79] Angeregt durch: Leibniz (Couturat, Fragm[ents] etc.) [...] [84] 7. Der Weg, die Elemente der Dinge zu finden (d. h. die prim[ae] not[iones]), besteht vielleicht darin, wenn möglich, den ,gemeinsamen Teiler“ je zweier Dinge zu finden (Eukl[idischer] Algorith$m[u s]$ ). Das wäre die Def[inition] der Elemente in terms of $\varepsilon$ (oder ,wahr“). Carnap [85] definiert die Elemente in terms of ,Ähnlichkeit“. ${ }^{55}$

Diese Bemerkung beginnt mit ,angeregt durch Leibniz“ und erstreckt sich dann über mehrere Manuskriptseiten, einschließlich der Passage, in der Gödel dann auf Carnaps Methode, die einfachen Elemente mittels Ähnlichkeit zu definieren, zu sprechen kommt. Interessant hieran ist nicht zuletzt, dass Gödel selbst Carnap in den Kontext seiner durch Leibniz angeregten Überlegungen stellt. Gödel scheint also bekannt zu sein, dass Leibniz auch für Carnaps Philosophie eine Vorbildfunktion hatte. ${ }^{56}$

Max Phil XI, 1944

[1] Bem[erkung] (Phil[osophie]), angeregt durch Carnap): ${ }^{57}$ Wir nehmen die Zeitpunkte und Raumpunkte [Richtungen, Entfernungen] unmittelbar wahr und ebenso die Relation der Erlebnisse zwischen diesen [nicht aber raumzeitliche Relationen der Erlebnisse untereinander]. Im Gegensatz dazu nehmen wir Ähnlichkeitsrelationen [oder Verhältnisse der Zusammensetzung] zwischen Farben wahr und nicht die Röte in der Farbe parametrisch. Also Raumzeit (obwohl außer uns objektiv existierend) wird doch (ohne besonderes Sinnesorgan dafür) unmittelbar wahrgenommen. Die Verhältnisse des Farbenraumes werden durch eine „,sinnliche Wesensschau“ wahrgenommen..$^{58}$ Dies schließt nicht aus, dass es uns unbekannte Farben gibt. Die „logische Wesensschau“ ist dasselbe auf höherer Stufe. Auch die Wahrnehmung der untersten Begriffe [z. B. Farbe] gehört zur Sinnlichkeit [nicht nur die Wahrnehmung von sinnlichen einzelnen Tatsachen].

\footnotetext{
${ }^{55} \mathrm{Zu}$, ̈̈hnlichkeit‘ bei Carnap siehe Carnap (1928, bes. S. 13, 109 f.).

${ }^{56}$ Awodey und Carus (2010) arbeiten sowohl Carnaps als auch Gödels Aspirationen, eine Characteristica universalis à la Leibniz zu begründen, heraus. Zu Carnap vgl. Awodey und Carus (2010, S. 252 f., 262, 266, 270 f.).

${ }^{57}$ Vgl. Carnap (1928, S. 120-127).

${ }^{58}$ Fußnote von Gödel dazu: „Es sind interne [essentielle] Verhältnisse, nicht äußere [accidentelle].“
} 
Diese Bemerkung ist insofern besonders aufschlussreich, als sie zeitlich kurz nach der langen Bemerkung ,angeregt durch Leibniz“ folgt. Auch wenn Carnaps Philosophie, anders als die von Leibniz, für Gödel inhaltlich keine Vorbildfunktion hat, lässt die wortgleiche Formulierung ,,angeregt durch“ in beiden Fällen erkennen, welche Bedeutung die Auseinandersetzung mit Carnaps Gedanken für Gödel angenommen hat, nachdem er diese nicht mehr nur aus rein logischem Interesse reflektiert hat.

Max Phil XI, 1944

[5] Carn[ap], Log[ische] Synt [ax], p. 313:59 An der Behauptung, dass die Übersetzbarkeit eine[r] phil[osophischen] Frage in die ,formal mode of speech" das Kriterium dafür ist, dass sie einen Sinn hat, ist insofern [etwas] richtig, als das wirkliche Kriterium das ist, ob es ein Vorschlag zu einer Theorie ist (oder Gegenüberstellung zweier Theorien), das heißt in der Absicht aufgestellt, eine Theorie vorzubereiten, oder in der Absicht, den Verstand zu verwirren und die Aufstellung einer Theorie zu verhindern. (Viele Philosophen kehren vielleicht ihre „Mission“ ebenso ins Gegenteil um wie die Priester.)

Gödel hat offensichtlich seine Lektüre von Carnaps Logische Syntax der Sprache in Princeton auf Englisch fortgesetzt. In diesem Zusammenhang beschäftigt er sich nicht nur mit der großen Debatte innerhalb des Wiener Kreises um das Sinnkriterium philosophischer Sätze, sondern insbesondere mit der Rolle der Syntax in Carnaps Philosophie. Wie lange Gödel sich mit diesem Thema auseinandergesetzt hat, wissen wir aus der posthumen Veröffentlichung von Gödels Aufsatz „Is mathematics syntax of language?" in mehreren Varianten. ${ }^{60}$

Das Verhältnis von inhaltlicher und formaler Rede bei Carnap ist auch das Thema in den beiden folgenden ausgewählten Bemerkungen:

Max Phil XII, 1944-1945

[8] Bem [erkung] (Phil[osophie]): Es sei ein System von allgemeinen Gesetzen gegeben (welche Eigenschaften und Beziehungen einer Anzahl von Dingen (Individuen) betreffen). Wie ist es dann möglich, die „Wirkende“ und „Leidende“, das „Hätte“ und eine „Verursachung[s]“-Relation zu definieren? Beispiel Fernwirkungsgesetz der Elektrodynamik und Fresnelsche[s] Prinzip (ähnlich der Carnapsche Versuch, aus einer formalen Regel der Sprache die Interpretation zu bestimmen).

Phil XV [1955-]

\footnotetext{
${ }^{59}$ In der englischen Fassung von Logische Syntax der Sprache von 1937 beginnt auf S. 302 der Abschnitt § 79: „Philosophical Sentences in the Material and the Formal Mode of Speech“. In der unveränderten zweiten deutschen Auflage von 1968 hat $§ 79$ die Überschrift: „Philosophische Sätze in inhaltlicher und formaler Redeweise“. Die syntaktischen Sätze werden in formaler Redeweise abgefasst. Interessant sind die Beispielsätze 33a und 33b: „33a. Dieser Umstand (oder: Sachverhalt, Vorgang, Zustand) ist logisch notwendig; ... logisch unmöglich (oder: undenkbar); ... logisch möglich (oder: denkbar).“ „33b. Dieser Satz ist analytisch; ... kontradiktorisch; ... nicht kontradiktorisch.“ Auf S. 313 heißt es: „Translatability into the formal mode of speech constitutes the touchstone for all philosophical sentences, or, more generally, for all sentences which do not belong to the language of any one of the empirical sciences."

${ }^{60}$ In Gödel (1995, S. 334-362).
} 
[20] Phil[osophie]: Auch bei der Carnapschen in[ner]mat[hematischen] Def[inition] können die mat[hematischen] Sätze einen Inhalt haben, aber alle denselben und den kleinstmöglichen. Das heißt vielleicht: Auch die Mathematik hat einen empirischen Inhalt, nämlich dass etwas existiert [21] (daher unzweckmäßig, die Logik ohne diese Annahme aufzubauen).

Zusammenfassend lässt sich zu Gödels Beschäftigung mit Carnap festhalten, dass dieser auf Gödels Arbeiten der Jahre 1930 und 1931 einigen Einfluss hatte. Das wurde in der Forschung bereits herausgearbeitet. Gödel und Carnap haben sich in dieser Periode allerdings gegenseitig vornehmlich als mathematische Logiker rezipiert. Bei Gödel ändert sich das später; für Carnap blieb Gödel vornehmlich jemand, der ihm half, seine logischen Probleme zu lösen. ${ }^{61}$ Ab 1934 beginnt Gödel, sich mit Carnap als Philosoph zu befassen; das zeigt auch sein Lektüreprogramm. Gödel sieht darüber hinaus eine gewisse Nähe von Carnaps Philosophie zu der von Leibniz, die für ihn selbst eine Vorbildfunktion hat, kritisiert aber Carnaps Philosophiekonzeption.

\section{Literatur}

Awodey, S., und A.W. Carus. 2001. Carnap, completeness, and categoricity: The Gabelbarkeitssatz of 1928. Erkenntnis 54:145-172.

- 2010. Carnap and Gödel. In Kurt Gödel: Essays for his centennial, Hrsg. S. Feferman, C. Parsons, and S.G. Simpson, 252-274. Cambridge: Cambridge University Press.

Carnap, R. 1928. Der logische Aufbau der Welt. Berlin/Schlachtensee: Weltkreis.

. 1931/32. Die logizistische Grundlegung der Mathematik. Erkenntnis 2:91-105.

1934. Logische Syntax der Sprache. Wien: J. Springer.

1937. The logical syntax of language. Übers. v. A. Smeaton. London: Routledge and Kegan Paul.

Carus, A.W. 2007. Carnap and twentieth-century thought: Explication as enlightenment. Cambridge: Cambridge University Press.

Dawson, J.W., Jr. 1999. Kurt Gödel: Leben und Werk. Wien/New York: Springer.

Gödel, K. 1986. Collected works, Bd. I: Publications 1929-1936, Hrsg. v. S. Feferman, J.W. Dawson Jr., S. Kleene, G.H. Moore, R.M. Solovay und J. van Heijenoort. Oxford: Oxford University Press.

- 1995. Collected works, Bd. III: Unpublished essays and lectures, Hrsg. v. S. Feferman, J.W. Dawson Jr., C. Parsons, W. Goldfarb, und R.M. Solovay. Oxford: Oxford University Press. . 2003a. Collected works, Bd. IV: Correspondence A-G., Hrsg. v. S. Feferman, J.W. Dawson Jr., W. Goldfarb, C. Parsons, und W. Sieg. Oxford: Clarendon Press.

. 2003b. Collected works, Bd. V: Correspondence H-Z., Hrsg. v. S. Feferman, J.W. Dawson Jr., W. Goldfarb, C. Parsons, und W. Sieg. Oxford: Clarendon Press.

. 2019. Philosophische Notizbücher/Philosophical Notebooks, Bd. 1: Philosophie I Maximen 0/Philosophy I Maxims 0, Hrsg. v. Eva-Maria Engelen. Berlin: De Gruyter.

\footnotetext{
${ }^{61}$ Ein abschließendes Urteil dazu wird allerdings erst die Transkription von Carnaps Tagebüchern der Jahre 1952-1954 erlauben.
} 
2020. Philosophische Notizbücher/Philosophical Notebooks, Bd. 2: Zeiteinteilung (Maximen) I und II/Time Management (Maxims) I and II, Hrsg. v. Eva-Maria Engelen. Berlin: De Gruyter.

Goldfarb, W. 2003. Rudolf Carnap. Gödel 2003a:335-341.

. 2005. On Gödel's way in: The influence of Rudolf Carnap. Bulletin of Symbolic Logic 11: 185-193.

Schimanovich-Galidescu, M.-E. 2002. Archivmaterial zu Kurt Gödels Wiener Zeit 1924-1940. In Kurt Gödel: Wahrheit und Beweisbarkeit, Bd. 1: Dokumente und historische Analysen, Hrsg. v. E. Köhler, P. Weibel, M. Stöltzner, B. Buldt, and C. Klein, 135-147. Wien: W. DepauliSchimanovich-Göttig. öbv \& hpt.

Stadler, F. 2015. Der Wiener Kreis: Ursprung, Entwicklung und Wirkung des Logischen Empirismus im Kontext. Wien: Springer.

Open Access This chapter is licensed under the terms of the Creative Commons Attribution 4.0 International License (http://creativecommons.org/licenses/by/4.0/), which permits use, sharing, adaptation, distribution and reproduction in any medium or format, as long as you give appropriate credit to the original author(s) and the source, provide a link to the Creative Commons license and indicate if changes were made.

The images or other third party material in this chapter are included in the chapter's Creative Commons license, unless indicated otherwise in a credit line to the material. If material is not included in the chapter's Creative Commons license and your intended use is not permitted by statutory regulation or exceeds the permitted use, you will need to obtain permission directly from the copyright holder.

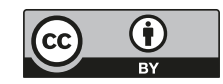

